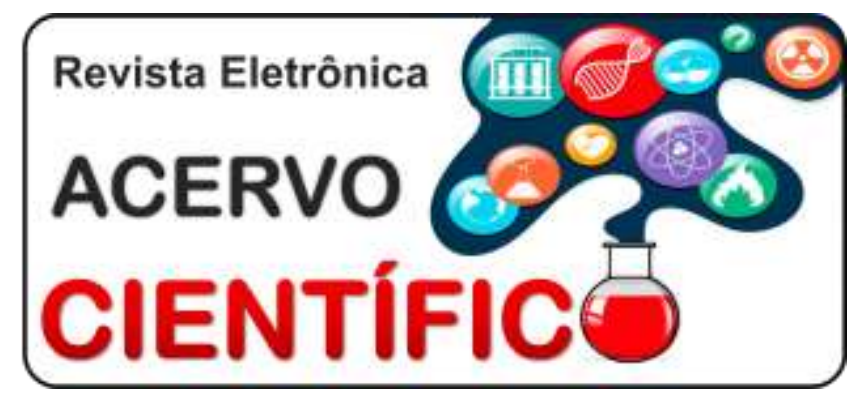

ARTIGO ORIGINAL

Recebido em: 10/2019

Aceito em: 11/2019

Publicado em: 12/2019

\title{
Validação de roteiro de sistematização da assistência de enfermagem à gestante na atenção primária
}

\author{
Validation of Systematization of Nursing care for pregnant care \\ Validación de la sistematización de la atención de enfermería para la atención del \\ embarazo
}

Luciana Batista ${ }^{1 *}$, Silvia Matumoto

Resumo: Esse artigo buscou evidenciar a importância da sistematização da assistência de enfermagem
como instrumento valioso no trabalho do enfermeiro, produzindo registro de suas práticas por meio de
processo organizado com linguagem padronizada. Objetivou reformular e validar roteiro para primeira
consulta de enfermagem à gestante de baixo risco utilizando a CIPESC através de estudo metodológico
realizado por validação de conteúdo por especialistas. O roteiro validado oferece subsídios para direcionar
os enfermeiros a documentarem a consulta de enfermagem, proporciona atendimento integral e
humanizado às gestantes, qualifica o atendimento, evita falhas na comunicação e possibilita intervir
oportunamente em alterações no período gestacional produzindo impacto nos índices de morbimortalidade
materna e infantil, além de proporcionar material para estudos relacionados à sua aplicabilidade.

Palavras-chave Cuidados de enfermagem, Atenção primária à saúde, Classificação internacional de atenção primária.

\begin{abstract}
This article sought to highlight the importance of systematizing nursing care as a valuable instrument in the work of nurses, producing a record of their practices through a process organized with standardized language. The objective was to reformulate and validate the script for the first nursing consultation for low-risk pregnant women using the CIPESC through a methodological study conducted by content validation by specialists. The validated script offers subsidies to direct nurses to document the nursing consultation, provides full and humanized care to pregnant women, qualifies care, avoids communication failures and enables timely intervention in changes in pregnancy, impacting maternal and child morbidity and mortality rates, besides providing material for studies related to its applicability.
\end{abstract}

Keywords: Nursing care, Primary health care, International classification of primary care.

Resumen: Este artículo buscaba resaltar la importancia de sistematizar la atención de enfermería como un instrumento valioso en el trabajo de las enfermeras, produciendo un registro de sus prácticas a través de un proceso organizado con lenguaje estandarizado. El objetivo era reformular y validar el guión para la primera consulta de enfermería para mujeres embarazadas de bajo riesgo utilizando el ICESCR a través de un estudio metodológico realizado por validación de contenido por expertos. El script validado ofrece subsidios a las enfermeras directas para documentar la consulta de enfermería, brinda atención completa y

${ }^{1}$ Escola de Enfermagem da USP de Ribeirão Preto (EERP/USP). Ribeirão Preto - SP. *E-mail: luciana_bat@hotmail.com 
humanizada a las mujeres embarazadas, califica la atención, evita fallas de comunicación y permite la intervención oportuna en los cambios en el embarazo, impactando las tasas de morbilidad y mortalidad materna e infantil, además de proporcionar material para estudios relacionados con su aplicabilidad.

Palabras clave: Cuidados de enfermería, Atención primaria de salud, Clasificación internacional de atención primaria.

\section{INTRODUÇÃO}

A Sistematização da Assistência de Enfermagem (SAE) tornou-se uma ferramenta indispensável ao processo do cuidado do enfermeiro (XAVIER LF, et al., 2018). Na prática clínica observa-se que o enfermeiro utiliza o processo de enfermagem (PE) como instrumento de trabalho, no entanto, o registro sistematizado deste, muitas vezes não é realizado adequadamente devido à falta de ferramentas, protocolos entre outros (RIBEIRO GC e PADOVEZE MC, 2018).

Além disso, sob a ótica da organização do trabalho em saúde, o uso de sistemas de classificação de enfermagem ainda constitui um desafio para os enfermeiros, sobretudo na saúde coletiva, seja pelo aumento da complexidade do processo saúde doença, da consolidação dos serviços de Atenção Primária (AP) como porta de entrada do Sistema Único de Saúde (SUS) ou mesmo pela falta de domínio de referenciais teóricos e metodológicos (RIBEIRO GC e PADOVEZE MC, 2018; SANTOS JRS e MURAI HC, 2010).

A SAE, regulamentada pela Resolução oㅜ 358/2009 do Conselho Federal de Enfermagem (COFEN), estabelece a implantação, planejamento, organização, execução e avaliação do processo de enfermagem privativamente pelo enfermeiro, em toda instituição de saúde, pública e privada (RIBEIRO GC e PADOVEZE MC, 2018) propondo resultados e melhorias efetivas da qualidade da assistência de enfermagem não visando apenas o indivíduo e sim todo o contexto em que ele está inserido (BRASIL, 2016).

É nessa visão que se faz necessário sistematizar o cuidado de enfermagem levando-se em conta as necessidades biológicas articuladas às sociais e ambientais, nas quais mesmo sendo distintas entre si têm suas origens na reprodução da vida em sociedade (GOMES LFS, 2017).

Na Atenção Primária está incutido na rotina do enfermeiro a consulta do pré-natal, e mesmo a gestação sendo um evento essencialmente fisiológico na vida da mulher a coloca no limite do patológico, uma vez que se caracteriza por várias e profundas transformações físicas e emocionais, exigindo um acompanhamento dos profissionais da saúde para evitar situações de risco para mãe e o concepto (GOMES LFS, 2017). Isto posto, o atendimento do enfermeiro, deve ser de forma integral e humanizado através de processo sistematizado visando atender as necessidades da mãe, seus familiares e o meio em que essa está inserida na comunidade (BRASIL, 2016).

A mortalidade materna é um indicador para avaliar as condições de saúde e de vida de uma população e ainda constitui um desafio para a saúde pública no prisma da assistência pré-natal (BRASIL, 2016). O atendimento do enfermeiro através da aplicação do processo de enfermagem possibilita o levantamento de problemas evitáveis tanto na gestação como no parto e puerpério, sendo uma forma de qualificar o atendimento através da integralidade, levantando diagnósticos e prescrevendo intervenções que possibilitam ações durante 0 pré-natal que podem proporcionar a diminuição dos índices de morbimortalidade materna (RIBEIRO GC e PADOVEZE MC, 2018).

Para tanto, faz-se necessário a escolha da classificação da assistência de enfermagem voltada para a avaliação da integralidade do ser humano no aspecto biopsicossocial e socioeconômico. A Classificação Internacional de Práticas de Enfermagem em Saúde Coletiva (CIPESC), projeto brasileiro, que se constitui de um inventário vocabular no campo da saúde coletiva que considerou a qualificação da força de trabalho em enfermagem expressando a prática ancorada na sociedade, grupos sociais, vida, saúde, doença e trabalho, sendo, portanto, a mais indicada para ser utilizada nesse grupo específico (MESQUITA SKC e RAMOS DKR, 2014). 
Logo a relevância do presente estudo se pauta na importância de elaborar um roteiro sistematizado, com fundamentação científica que alcance de uma assistência qualificada na primeira consulta de pré-natal na Atenção Primária. Destarte, o objetivo desse estudo foi validar roteiro para primeira consulta de enfermagem à gestante na Atenção Primária, utilizando a CIPESC.

\section{MÉTODOS}

Estudo metodológico por Validação de Conteúdo em processo de desenvolvimento e aperfeiçoamento de um instrumento que possa aprimorar uma metodologia utilizando como estratégia o conhecimento existente para elaboração de uma nova intervenção ou melhora significativa da intervenção existente, validando-o pela importância para o público-alvo através do julgamento de diferentes examinadores especialistas, que analisam a representatividade dos itens em relação às áreas de conteúdo e à relevância dos objetivos a medir consistindo em um importante critério para avaliação de um roteiro, definindo desta forma, se este roteiro está apropriado para atingir o objetivo proposto (RIBEIRO MAS, et al., 2013).

O ponto de partida do estudo foi o roteiro elaborado, pelos enfermeiros de uma Unidade Básica de Saúde do interior do estado de São Paulo, para atender às demandas de primeira consulta de gestantes, a partir de conhecimentos práticos, sem observar critérios de método científico.

Para aprimorar o roteiro foram realizadas buscas em três bases de dados científicas: Medical Literature Analysis and Retrieval System Online (MEDLINE), Scientific Electronic Library Online (SCIELO) e Literatura Latino-Americana e do Caribe em Ciências da Saúde (LILACS), limitando a artigos primários inseridos em um período de 10 anos (2005 a 2016) em inglês, português e espanhol; e foram utilizados os descritores Atenção primária, gestante, pré-natal, sistematização da assistência de enfermagem e cuidados de enfermagem.

Após a realização da busca, foram selecionados os estudos primários através da leitura dos títulos e dos resumos, seguindo os critérios de inclusão: artigos de estudos primários que abordavam o tema classificação e sistematização da assistência de enfermagem à gestante na $A B$; e os critérios de exclusão adotados foram: produção duplicada, teses e dissertações, artigos de revisão, de opinião e ensaios teóricos, cartas ao editor, boletins e resumos.

Os artigos selecionados para revisão foram submetidos à leitura criteriosa, identificando e agrupando variáveis de caracterização socioeconômicas e biológicas, diagnósticos e intervenções de enfermagem em instrumento formulado pela autora para este estudo. Também foi realizado estudo e análise aprofundada da CIPESC. Após esse processo reformulou-se o roteiro inserindo-se variáveis complementares às existentes que possibilitassem a caracterização e a identificação de problemas evitáveis na gestação, assim como diagnósticos de enfermagem e intervenções que não estavam contempladas no roteiro inicial.

Para o processo de validação, o novo roteiro foi dividido em itens, nos quais cada variável, diagnóstico e intervenção recebeu uma numeração, totalizando cento e onze itens para apreciação da comissão de especialistas.

Para compor a comissão foram selecionados intencionalmente, através dos dados encontrados nos artigos primários, quarenta e sete profissionais para serem convidados a participar do estudo. Os profissionais atenderam os seguintes critérios de inclusão: ser enfermeiro (a); ter experiência na Atenção Primária ou ter estudado o tema; possuir conhecimento e experiência com a CIPESC. Convencionou-se de acordo com referência literária entre cinco e dez especialistas para compor a equipe mínima de especialistas (ALEXANDRE NM e COLUCI MZO, 2011).

O contato inicial deu-se por e-mail. Para todos foram enviados e-mails com a descrição e convite para participar do estudo. Dentre os quarenta e sete convidados, doze responderam positivamente aceitando participar do estudo; cinco recusaram, apresentando como justificativa não estar mais atuando na área de estudo; dezesseis não responderam ao e-mail e os outros quatorze os endereços eletrônicos indexados nos artigos não existia. 
Todos os especialistas que aceitaram o convite optaram para receber o material via e-mail; desta forma, foi enviado o Termo de Consentimento Livre e Esclarecido (TCLE), ficha de identificação dos especialistas e o instrumento para apreciação. A ficha de identificação dos juízes continha dados tais como o nome, idade, tempo de experiência, área de atuação (assistência ou docência) e se já havia participado de outro estudo de validação de instrumento/roteiros.

As avaliações de apenas oito juízes retornaram após dois meses com a análise dos itens, sugestões de alteração, acréscimo e a caracterização dos mesmos. Estipulou-se segundo Viana VO e Pires PSP (2014) a seguinte pontuação: +1 (adequado), 0 (impreciso) e -1 (inadequado), sendo considerados validados os itens que receberem aprovação de pelo menos $75 \%$ dos especialistas. Não foi estipulada a quantidade de apreciações para a validação. A validação aconteceu em duas rodadas, no período de fevereiro a junho de 2017.

O projeto foi submetido e aprovado pelo Comitê de Ética em Pesquisa da Escola de Enfermagem de Ribeirão Preto da Universidade de São Paulo (USP) no: 60761816.7.0000.5393, e pela Comissão de Avaliação de Projetos de Pesquisa da Secretaria Municipal de Saúde da Prefeitura Municipal de Ribeirão Preto, através do Ofício 3524/16-GS, conforme a Resolução 466/2012 do Conselho Nacional de Saúde (CNS, 2012).

\section{RESULTADOS}

A comissão de juízes foi composta por oito membros, com predomínio do sexo feminino, com mais de 30 anos de idade, com mais de 10 anos de graduação em enfermagem, com pós-graduação nível doutorado, atuantes nas regiões Sudeste e Nordeste do país e que participaram como avaliadores em outros estudos. O detalhamento da caracterização desses especialistas segue apresentada na Tabela 1.

Tabela 1 - Caracterização da Comissão de Juízes.

\begin{tabular}{|c|c|c|c|}
\hline \multicolumn{2}{|c|}{ Caracterização da comissão de Juízes } & \multirow{2}{*}{$\begin{array}{l}\mathbf{N} \\
1 \\
7 \\
\end{array}$} & \multirow{2}{*}{$\begin{array}{c}\% \\
12,5 \\
87,5 \\
\end{array}$} \\
\hline $\operatorname{Sexo}(n=8)$ & $\begin{array}{l}\text { Masculino } \\
\text { Feminino }\end{array}$ & & \\
\hline Faixa etária $(\mathrm{n}=8)$ & $\begin{array}{l}20-30 \text { anos } \\
31-40 \text { anos } \\
41-50 \text { anos } \\
+50 \text { anos }\end{array}$ & $\begin{array}{l}1 \\
4 \\
1 \\
2\end{array}$ & $\begin{array}{l}12,5 \\
50,0 \\
12,5 \\
25,0\end{array}$ \\
\hline Tempo de graduação em enfermagem $(n=8)$ & $\begin{array}{r}1-10 \text { anos } \\
11-20 \text { anos } \\
21-30 \text { anos }\end{array}$ & $\begin{array}{l}1 \\
5 \\
2\end{array}$ & $\begin{array}{l}12,5 \\
62,5 \\
25,0\end{array}$ \\
\hline Cursos de Pós-Graduação (n=8) & $\begin{array}{l}\text { Especialização } \\
\text { Mestrado } \\
\text { Doutorado }\end{array}$ & $\begin{array}{l}1 \\
2 \\
5\end{array}$ & $\begin{array}{l}12,5 \\
25,0 \\
62,5\end{array}$ \\
\hline Área de atuação (n=8) & $\begin{array}{l}\text { Atenção Primária } \\
\text { Docência universitária } \\
\text { Atenção Primaria + Docência }\end{array}$ & $\begin{array}{l}2 \\
5 \\
1\end{array}$ & $\begin{array}{l}25,0 \\
62,5 \\
12,5\end{array}$ \\
\hline Região do país em que atua $(n=8)$ & $\begin{array}{l}\text { Sudeste } \\
\text { Nordeste }\end{array}$ & $\begin{array}{l}4 \\
4\end{array}$ & $\begin{array}{l}50,0 \\
50,0\end{array}$ \\
\hline Publicação sobre temática (n=8) & $\begin{array}{l}1 \text { publicação } \\
2 \text { a } 5 \text { publicações } \\
+20 \text { publicações - } 1\end{array}$ & $\begin{array}{l}1 \\
1 \\
1\end{array}$ & $\begin{array}{l}33,3 \\
33,3 \\
33,3\end{array}$ \\
\hline $\begin{array}{l}\text { Participação como juiz em outros estudos } \\
(\mathrm{n}=8)\end{array}$ & $\begin{array}{l}\text { Sim } \\
\text { Não }\end{array}$ & $\begin{array}{l}6 \\
2\end{array}$ & $\begin{array}{l}75,0 \\
25,0\end{array}$ \\
\hline
\end{tabular}

Fonte: Batista L, Matumoto S, 2017. 
O roteiro inicial do estudo continha 53 itens, distribuídos em 3 grupos: entrevista e exame físico, Diagnóstico de enfermagem e Intervenções de enfermagem. A partir da revisão da literatura a versão reestruturada ficou com 111 itens. Esta versão foi analisada pela comissão de juízes, cuja primeira etapa ocorreu em fevereiro de 2017 e os resultados constam da Tabela 2.

Tabela 2 - Apreciação pela Comissão de juízes da versão reestruturada do roteiro de sistematização da assistência de enfermagem da primeira consulta de pré-natal.

\begin{tabular}{lcc}
\hline Avaliação dos itens & $\mathbf{n}$ & $\%$ \\
\hline Aprovado $100 \%$ & 48 & 43,6 \\
Aprovado $75 \%$ & 42 & 38,2 \\
Impreciso ou inadequado & 20 & 18,2 \\
\hline Total de itens & 110 & 100 \\
\hline
\end{tabular}

Fonte: Batista L, Matumoto S, 2017.

As sugestões dos avaliadores na primeira apreciação foram: acréscimo de data de nascimento; mudança de profissão para ocupação; imprecisão no item peso anterior; mudança de estado civil para condição de união; inclusão da última gestação; adequação do estado nutricional de acordo com as normatizações do Ministério da Saúde (MS) relacionando o peso com idade gestacional e IMC (Índice de Massa Corpórea); no item antecedentes ginecológicos foi sugerida a descrição de acontecimentos prévios, inclusão de cirurgia anterior; substituição de interação social por suporte social; inclusão descrição da atividade física e atividade de laser. Quanto às vulnerabilidades, fatores de risco, medicações em uso especificar quais estariam expostas e ou utilizando. Quanto ao exame físico, as sugestões foram de contemplar todos os possíveis achados não apenas os alterados.

No tópico Diagnostico de Enfermagem (DE), praticamente todos os itens sofreram sugestões, ora por ter apenas a normalidade, ora por apresentar somente as alterações, de forma que as sugestões foram adequá-los com as duas formas de padrão. Também foi sugerida a incorporação dos DE: medo e conforto.

Nas intervenções de enfermagem, foram sugeridos: correções do tempo verbal e acrescentar o verbo "orientar" em alguns itens; verificação de itens que se relacionavam a atividades administrativas do enfermeiro e não a intervenções; avaliar itens que contemplavam a mesma orientação, de forma diferenciada; acrescentar o período e data de retorno; melhorar a descrição de algumas orientações; acrescentar demais trimestres gestacionais, uma vez que o pré-natal pode ser iniciado além dos primeiros 120 dias.

Como resultado, o roteiro reformulado de acordo com as sugestões dos juízes e literatura passou a conter 113 itens, discriminados no Quadro 1.

Quadro 1 - Itens da $2^{\text {a }}$ versão do roteiro de SAE da primeira consulta de pré-natal

\begin{tabular}{|l|c|}
\hline \multicolumn{1}{|c|}{ Itens } & n \\
\hline Itens aprovados pelos juízes na 1ª apreciação (branco) & 75 \\
\hline $\begin{array}{l}\text { Itens que obtiveram 75\% de aprovação e foram reformulados conforme } \\
\text { sugestão dos juízes (cinza) }\end{array}$ & 16 \\
\hline Itens reformulados conforme sugestão dos juízes (amarelo) & 14 \\
\hline Itens novos sugeridos pelos juízes (laranja) & 8 \\
\hline Total & 113 \\
\hline
\end{tabular}

Fonte: Batista L, Matumoto S, 2017.

Uma vez reestruturado o formulário, iniciou-se em abril de 2017 a segunda rodada de apreciação, com foco em um total de 23 itens entre os novos e reestruturados, destacado em cores diferentes para melhor reavaliação dos juízes (Quadro 1). Em junho de 2017 o instrumento foi aprovado pelos juízes, sendo que 13 itens obtiveram $100 \%$ de aprovação e nove obtiveram $75 \%$. As principais sugestões nesta rodada estão descritas no Quadro 2. 
Quadro 2 - Sugestões finais e modificações.

\begin{tabular}{|l|l|}
\hline Item & Sugestão \\
\hline Drogadição & Substituição por uso de drogas \\
\hline Situação vacinal inadequada & Substituição por atrasada \\
\hline \multicolumn{2}{|c|}{ Espaço para anotações complementares } \\
\hline
\end{tabular}

Fonte: Batista L, Matumoto S, 2017.

O instrumento final ficou constituído de três conjuntos de itens, entrevista e exame físico, diagnóstico de enfermagem e intervenções de enfermagem, totalizando 113 itens.

\section{DISCUSSÃO}

A assistência ao pré-natal objetiva a promoção da saúde da gestante e do feto. Sua proposta visa a identificar as situações de risco para ambos, permitindo intervenções oportunas, com impacto direto nos índices de mortalidade materna e infantil, prematuridade e baixo peso ao nascer (GOMES LFS, 2017).

O pré-natal na Atenção Primária é realizado pela equipe multiprofissional. Cabe ao enfermeiro papel relevante nesse atendimento, sendo atribuição desse profissional realizar o diagnóstico de gravidez e iniciar o pré-natal, tornando-se responsável pela primeira consulta da gestante (VIANA VO e PIRES PSP, 2014). O enfermeiro possui embasamento teórico e científico para acompanhamento da gestante de baixo risco e representa um diferencial no atendimento qualificado (BRASIL, 2016; RIBEIRO GC e PADOVEZE MC, 2018; SOARES MI, 2015).

Os enfermeiros vêm buscando atingir a integralidade e humanização do atendimento às gestantes, utilizando a SAE como instrumento para apoiar esse objetivo (PRIMO CC, et al., 2015; ALVES CN, et al., 2013).

A SAE representa uma forma de autonomia do enfermeiro, que permite aproximação junto ao paciente, tanto no momento da sua elaboração quanto na prestação de cuidados futuros (SPAZAPAN MP, 2017). Da mesma forma, permite o desenvolvimento de seu potencial intelectual, amplia sua atuação e tomada de decisão considerando a qualidade e o comprometimento com a humanização da assistência. Desta forma, o enfermeiro consolida seu papel social, sustentado pela melhora da qualidade dos registros (SPAZAPAN MP, 2017; MESQUITA SKC e RAMOS DKR, 2014).

É notório para a realização da SAE novos referenciais, ampliando o campo de visão para além das fórmulas prescritivas e normativas, adequando a assistência instituída no ser humano de forma individual e integral (CASTRO RR, et al., 2016).

Desta forma, o roteiro sistematizado auxilia e traz benefícios a esse atendimento norteando as ações do profissional, identificando as individualidades da gestante proporcionando 0 atendimento de suas necessidades de saúde na perspectiva da saúde como direito.

Foi possível identificar a importância e a fragilidade da SAE no âmbito da AP. Os achados fortaleceram a ideia de que a anamnese da gestante é fator determinante da qualidade do atendimento e representa a identificação de fatores de risco e de alerta na gestação, produzindo um forte impacto na diminuição dos índices de morbimortalidade (ALVES CN, et al., 2013; MESQUITA SKC e RAMOS DKR, 2014).

Os achados neste estudo trouxeram a necessidade de identificar as gestantes inseridas em seus meios sociais, econômicos e demográficos, a fim de estabelecer o levantamento de diagnósticos focados na individualidade da pessoa ao invés da associação focalizada em patologias, uma vez que quando se pensa em gestação, poucas vezes existe relação a elas. A entrevista e a anamnese foram pontuados como primordial para a atingir a integralidade no atendimento e adequá-los a proposta do Programa de Humanização no Pré-natal e Nascimento (PHPN), evitando esquecimentos e falhas, aprimorando técnicas e condutas, visando proporcionar assistência humanizada. 
Corroborando com esses achados, uma pesquisa realizada em Porto Alegre, Rio Grande do Sul, em 2013, traçou o perfil epidemiológico das gestantes de uma determinada unidade de AP localizada em situação de vulnerabilidade social.

O estudo identificou as mesmas variáveis presentes neste estudo, denotando a importância da coleta desses dados em diferentes regiões do país. Identificou-se que através de uma anamnese qualificada podese avaliar desde o perfil da clientela, adaptar os programas e protocolos existentes, desenvolver assistência de qualidade, humanizada com integralidade, assim como, avaliar a assistência prestada pela equipe (GOMES MRT e CÉSAR JA, 2013).

Mais recentemente, em 2015, com o intuito de avaliar a obesidade em mulheres brasileiras associadas à paridade, foi desenvolvido um estudo com o propósito de coletar variáveis socioeconômicas, que representou um agente modificador na qualidade do atendimento. Essa pesquisa teve representação das cinco macrorregiões brasileiras, das áreas urbanas e rurais, com um total de 15.575 mulheres entrevistadas. O estudo corrobora o poder modificador da assistência prestada quando a equipe avalia e conhece sua população, podendo prestar assistência qualificada, através dos dados subsidiados para aprimorar sua atuação como equipe (FERREIRA RAB e BENICIO MHD'A, 2015).

Quanto aos diagnósticos de enfermagem, a CIPESC não teve representatividade nas publicações atuais, mesmo sendo a mais indicada para atender a população na AP (MESQUITA SKC e RAMOS DKR, 2014; GOMES MRT e CÉSAR JA, 2013). Em um estudo de 2014 sobre a utilização da CIPESC foi demonstrado que em 2008, nas bases de dados inseridas na Biblioteca Virtual em Saúde, menos de dez por cento dos estudos científicos sobre Classificação de Enfermagem se referiam a essa classificação (CAVALCANTE MDMA, 2014).

O roteiro para subsidiar as consultas de enfermagem tem como potência proporcionar melhorias na qualidade do atendimento prestado à gestante, contribuir para o levantamento de dados para futuros estudos acerca da qualidade dos atendimentos, organização da dinâmica de trabalho do profissional de enfermagem, reconhecimento profissional, adequabilidade dos registros e atendimento das exigências do COFEN e dos Conselhos Regionais de Enfermagem (CORENs).

No entanto, é imprescindível que, na utilização do roteiro, o enfermeiro associe em seu manejo o uso de tecnologias leves relacionais que propiciem a interação com a gestante, como elemento essencial da humanização do cuidado.

Como viés, faz-se oportuno pontuar a quantidade escassa de artigos relacionados à primeira consulta de enfermagem no pré-natal e início da gestação. As publicações de forma geral enfocam as patologias associadas, preconizações do PHPN, parto, puerpério e amamentação (RIBEIRO GC e PADOVEZE MC, 2018).

Outro viés identificado pelo estudo é o número reduzido de artigos relacionados ao uso da CIPESC na literatura científica (CAVALCANTE MDMA, 2014) assim como falhas nos registros de enfermagem. Mesquita SKC e Ramos DKR (2014) corroboram esses dados em seus estudos, identificado a escassez de artigos sobre a temática na Atenção Primária.

\section{CONCLUSÃO}

Conclui-se que a Sistematização da Assistência de Enfermagem organiza e gerencia o atendimento de enfermagem, subsidia a avaliação de forma organizada das necessidades de saúde da pessoa em sua integralidade, não apenas visando à problemática que a fez buscar atendimento, envolvendo as esferas sociais, biológicas, econômicas e ambientas, proporcionando visão global do ser humano. A SAE qualifica o atendimento à gestante, possibilitando coletar informações identificando riscos e possibilita intervenções rápidas evitando assim, danos preveníeis ao binômio. O roteiro da SAE proposto facilita o cotidiano dos enfermeiros, diminui falhas na comunicação e a ausência de registro do cuidado prestado, além de servir como documento formal dos atendimentos e gerar oportunidade de diversos estudos sobre a temática abordada. 


\section{REFERÊNCIAS}

1. ALEXANDRE NM, COLUCI MZO. Validade de Conteúdo nos Processos de Construção e Adaptação de Instrumentos de Medidas. Revista Ciência \& Saúde Coletiva, 16 (7): 3061-3068, 2011.

2. ALVES CN et al. Perfil da gestante assistida no pré-natal de enfermagem de uma unidade Básica de Saúde. Revista de Pesquisa: Cuidado é Fundamental Online, Rio de Janeiro, v. 5, n. 3, p. 132-41, 2013.

3. BRASIL. Ministério Da Saúde. Protocolos da Atenção Básica: Saúde das Mulheres. Ministério da Saúde, Instituto Sírio-Libanês de Ensino e Pesquisa - Brasília: Ministério da Saúde, 2016.230 p.

4. CAVALCANTE MDMA. A Classificação Internacional para as Práticas de Enfermagem em Saúde Coletiva (CIPESC®) como instrumento do processo de trabalho do enfermeiro em saúde coletiva. 2014. 96 f. Dissertação (Mestrado) - Universidade Federal do Paraná, Curitiba, 2014.

5. CASTRO RR, et al. Compreensões e desafios acerca da sistematização da assistência de enfermagem, Rev enferm UERJ, Rio de Janeiro, 2016; 24(5): e10461.

6. CNS. CONSELHO NACIONAL DE SAÚDE. Resolução no 466, de 12 de dezembro de 2012.

7. FERREIRA RAB, BENICIO MHD'A. Obesidade em mulheres brasileiras: associação com paridade e nível socioeconômico. Revista Panamericana de Salud Pública, Washington, v. 37, n. 4-5, p. 337-42, 2015.

8. GOMES RMT, CÉSAR JA. Perfil epidemiológico de gestantes e qualidade do pré-natal em unidade básica de saúde em Porto Alegre, Rio Grande do Sul, Brasil. Revista Brasileira de Medicina de Família e Comunidade, Rio de Janeiro, v. 8, n. 27, p. 80-9, 2013.

9. GOMES LFS. Sistematização da Assistência de Enfermagem à gestante de alto risco: construção e validação de uma tecnologia para o cuidado. [TESE]. Fortaleza: Universidade Federal do Ceará; 2017.

10. MESQUITA SKC, RAMOS DKR. Produção científica sobre a classificação internacional das práticas de enfermagem em saúde coletiva. Revista da Universidade Vale do Rio Verde, Três Corações, v. 12, n. 2, p. 187-95, 2014.

11. MESQUITA SKC, RAMOS DKR. Produção científica sobre a classificação internacional das práticas de enfermagem em saúde coletiva. Revista da Universidade Vale do Rio Verde. 2014; 12 (2): 187-95.

12. PRIMO CC, et al. Classificação internacional para a prática de enfermagem na assistência pré-natal. Revista Enfermagem em Foco. 2015

13. RIBEIRO MAS, et al. Estudos de validação na enfermagem: revisão integrativa. Revista Rene, v. 14, n. 1, p. 21828, 2013.

14. RIBEIRO GC, PADOVEZE MC. Nursing Care Systematization in a basic health unit: perception of the nursing team. Rev Escol Enferm USP. 2018; 52.

15. SANTOS JRS, MURAI HC. Metodologia e Instrumentos para a Implantação da Sistematização da Assistência de Enfermagem na Atenção Básica. Revista de Enfermagem UNISA, v. 11, n. 1, p. 43-7, 2010.

16. SOARES MI, et al. Sistematização da assistência de enfermagem: facilidades e desafios do enfermeiro na gerência da assistência. Esc. Anna Nery. 2015 Mar; 19(1): 47-53.

17. SPAZAPAN MP. Processo de enfermagem na atenção primária: percepção de enfermeiros de Campinas-SP. Campinas, SP: [s.n.], 2017.

18. VIANA VO, PIRES PSP. Validação de instrumento de sistematização da assistência de enfermagem. Revista de Enfermagem e Atenção à Saúde. 2014; 3 (2): 64-75.

19. XAVIER LF, et al. Sistematização da Assistência de Enfermagem: o conhecimento de Enfermeiros do município de Ji-Paraná, Rondônia, Brasil. Rev. Nursing. 2018; 21 (239): 2110-2113. 